\title{
Una nueva localidad fosilífera en Oaxaca (México) y el registro más austral de Bison latifrons. Implicaciones paleobiogeográficas, paleoecológicas y paleoambientales
}

\author{
Roberto Díaz-Sibaja, Eduardo Jiménez-Hidalgo, Ma. Luisa García-Zepeda
}

\section{Roberto Díaz-Sibaja}

roberto.diaz.sibaja@gmail.com

Ma. Luisa García-Zepeda

Laboratorio de Paleontología, Facultad de Biología, Universidad Michoacana de San Nicolás de Hidalgo, Av. Francisco J. Múgica S/N, C.P. 58040, Morelia, Michoacán, México.

\section{Eduardo Jiménez-Hidalgo}

Laboratorio de Paleobiología, Instituto de Recursos, Universidad del Mar, campus Puerto Escondido, km 2.5 Carretera Puerto Escondido-Sola de Vega, C.P. 71980, Puerto Escondido, Oaxaca, México.
BOL. SOC. GEOL. MEX. 2018

VOL. 70 NO. 1

P. $201-222$

http://dx.doi.org/10.18268/BSGM2018v70n1a12

\section{RESUMEN}

Se reporta el registro más austral de Bison latifrons para América del Norte. El registro procede de una nueva localidad fosilífera en el municipio de San Dionisio Ocotepec, localizado en los Valles Centrales de Oaxaca. La fauna asociada incluye a Mammuthus columbi, Equus cf. E. conversidens y Bison sp. Por la presencia de bisontes y de mamut, se infiere una edad correspondiente al Rancholabreano (Pleistoceno tardío). Adicionalmente, se llevó a cabo un análisis de mesodesgaste para proboscídeos y un análisis de microdesgaste de baja magnificación (35x) a los restos dentales de mamut. Los resultados de estos análisis sugieren que el individuo de $M$. columbi era un pacedor. Debido a los hábitos dietarios del mamut y del hábitat inferido para caballos y bisontes de México, inferimos que, durante el Pleistoceno, en el sitio debieron existir zonas abiertas con pastizales en las partes planas. El presente registro de Bison latifrons es el primero de la especie para Oaxaca (México) y para la provincia morfotectónica del Cinturón Volcánico Transmexicano; con ello, su rango de distribución geográfica conocida se extiende en más de $447 \mathrm{~km}$, desde Tequixquiac en el Estado de México hasta el centro de Oaxaca. Esta nueva localidad fosilífera contribuye al conocimiento de las faunas del Pleistoceno tardío de la porción sur de México y en particular del centro de Oaxaca, lo que mejora nuestro entendimiento de los cambios bióticos y climáticos ocurridos durante el inicio del Holoceno.

Palabras clave: Rancholabreano, Oaxaca, Bison, Mammuthus, Equus.

\section{ABSTRACT}

We report the southernmost record of Bison latifrons in North America. This is the first record for the species in Oaxaca (Mexico), and for the Transmexican Volcanic Belt morphotectonic province; the known geographic distribution range of Bison latifrons is extended in more than $447 \mathrm{~km}$, from $T_{e}$ quixquiac in the State of Mexico to central Oaxaca. This finding comes from a new fossiliferous locality at the municipality of San Dionisio Ocotepec, located in the Central Valleys region of the state of Oaxaca, Mexico. The associated fauna includes: Mammuthus columbi, Equus cf. E. conversidens, and Bison sp. By the presence of bison as well as mammoth, a Rancholabrean land mammal age (Late Pleistocene) is assigned to the fossil fauna. In addition, we performed a proboscidean mesowear analysis as well as a low magnification microwear analysis (35x) to the dental elements of the mammoth. Our findings suggest that the mammoth individual was a grazer. Due to the mammoth's feeding habits and the habitat described for Mexican samples of bison and horses, we concluded that during the Late Pleistocene, San Dionisio Ocotepec was dominated by open grassland in the lowlands. This new fossil site and its fauna contribute to improve the knowledge of the Rancholabrean in southern Mexico, and particularly in the Central Valleys of Oaxaca. This improves our understanding of the biotic and climatic changes during the foundation of the Holocene.

Keywords: Rancholabrean, Oaxaca, Bison, Mammuthus, Equus. 


\section{Introducción}

El género Bison tiene una historia evolutiva corta y compleja. Evolucionó en Indochina durante el Plio-Pleistoceno, entre hace 3.4 y 2.6 Ma (Kurtén, 1968; McDonald, 1981; Khan et al., 2010; Castaños et al., 2012). Los bisontes se dispersaron por Eurasia hace aproximadamente $700 \mathrm{Ka}$, durante el Pleistoceno medio y arribaron a Beringia entre hace 300 y $130 \mathrm{Ka}$ (Shapiro et al., 2004). Finalmente, invadieron América del Norte continental entre hace 160 y $75 \mathrm{Ka}$ (McDonald, 1981; Haynes, 1985; Bell et al., 2004; Shapiro et al., 2004), donde evolucionaron dos especies autóctonas: Bison antiquus (syn $=$ B. occidentalis, sensu McDonald, 1981; Wilson et al., 2008) y Bison latifrons (Guthrie, 1970). Estas dos especies de bisontes se presentan de forma simpátrica con dos especies de origen eurasiático: B. priscus y B. alaskensis (Guthrie, 1970; McDonald, 1981). Todas estas especies coexistieron durante el Pleistoceno tardío de América del Norte, incluyendo México (McDonald, 1981; Ferrusquía-Villafranca et al., 2010), con excepción de B. bison, que se originó durante el Holoceno temprano $(4-5 \mathrm{Ka})$ en el sur de Canadá (McDonald, 1981; Wilson et al., 2008).

$\mathrm{El}$ bisonte gigante (B. latifrons) es uno de los bisontes menos conocidos de México. Se tienen registros de la especie en San Juan de los Lagos (Dugès, 1894; Solorzano, 2002), Zacoalco ( Solorzano, 2002; Lucas, 2008) y Chapala, Jalisco (McDonald, 1981; Solorzano, 2002); la Piedad-Santa Ana y la Cinta-Portalitos, Michoacán-Guanajuato (Díaz-Sibaja et al., 2012); Zumpango de Ocampo (Osborn, 1905) y Tequixquiac, Estado de México (Cope, 1884; Villada, 1903) y dos localidades sin nombrar en Zacatecas (Skinner y Kaisen, 1947). En Oaxaca sólo se tienen registros probables en la Mixteca alta, al norte del estado y en los Valles Centrales (Ferrusquía-Villafranca, 1976; Ferrusquía-Villafranca et al., 2010).

Los bisontes del Pleistoceno formaron parte de un ensamblaje mastofaunístico diverso, de cerca de 286 especies reportadas (Arroyo-Cabrales et al., 2002; Ferrusquía-Villafranca et al., 2010).
Esta riqueza de especies es comparable a la actual, aunque la composición y estructura de las comunidades es distinta (Ceballos et al., 2010; Ferrusquía-Villafranca et al., 2010; Ceballos y Arroyo-Cabrales, 2012). La mayoría de los registros de mamíferos cuaternarios en México corresponden a la NALMA (North American Land Mammal Age, edad de mamíferos terrestres norteamericanos) del Rancholabreano (Arroyo-Cabrales et al., 2002), la cual se caracteriza por la presencia del taxón índice Bison y corresponde al Pleistoceno tardío (160 - 9.5 Ka) (Savage, 1951; Bell et al., 2004). Durante el Rancholabreano se presentaron cambios climáticos importantes como por ejemplo, el Último Máximo Glaciar y el Dryas Reciente (Haynes, 2008; Clark et al., 2009). Estos cambios alteraron la composición de las comunidades mastofaunísticas, lo que se toma como referencia para establecer los inicios del Holoceno (Ceballos et al., 2010). El estudio de las faunas correspondientes al Rancholabreano contribuye al entendimiento de la fundación del Holoceno y de la respuesta de las comunidades de mamíferos al cambio climático (Arroyo-Cabrales et al., 2008).

Las faunas del Pleistoceno tardío en Oaxaca corresponden en su mayoría a la región de la Mixteca alta, localizada al noroeste del estado (Pérez-Crespo et al., 2008; Jiménez-Hidalgo et al., 2011) y a la región de los Valles Centrales (Pérez-Crespo et al., 2008). En este trabajo presentamos el registro más austral de Bison latifrons, su fauna asociada y el análisis de dieta de un ejemplar de mamut, procedente de una nueva localidad fosilífera del Rancholabreano en los Valles Centrales de Oaxaca.

\section{2. Área de estudio}

San Dionisio Ocotepec se localiza en el municipio del mismo nombre, al este de los Valles Centrales de Oaxaca. La localidad se encuentra entre las coordenadas $16^{\circ} 48^{\prime}-16^{\circ} 47^{\prime} \mathrm{N}$ y $96^{\circ} 24^{\prime}-$ $96^{\circ} 22^{\prime} \mathrm{O}$, con afloramientos fosilíferos en un par de arroyos de flujo intermitente denominados "El Pedregal” y "La Salina". La fisiografía de la zona 
es heterogénea, con presencia de zonas planas utilizadas con fines agrícolas y ganaderos, así como una amplia representatividad de arroyos y cañadas (Figura 1).

El yacimiento se localiza en una zona con aluvión
Cuaternario que sobreyace a tobas andesíticas y andesitas del Neógeno y secuencias de areniscas y lutitas de la Formación Teposcolula-Ocotlán (Cenomaniano-Albiano) y sólo en el sureste del sitio se encuentran calizas y lutitas de la Formación Yucu-

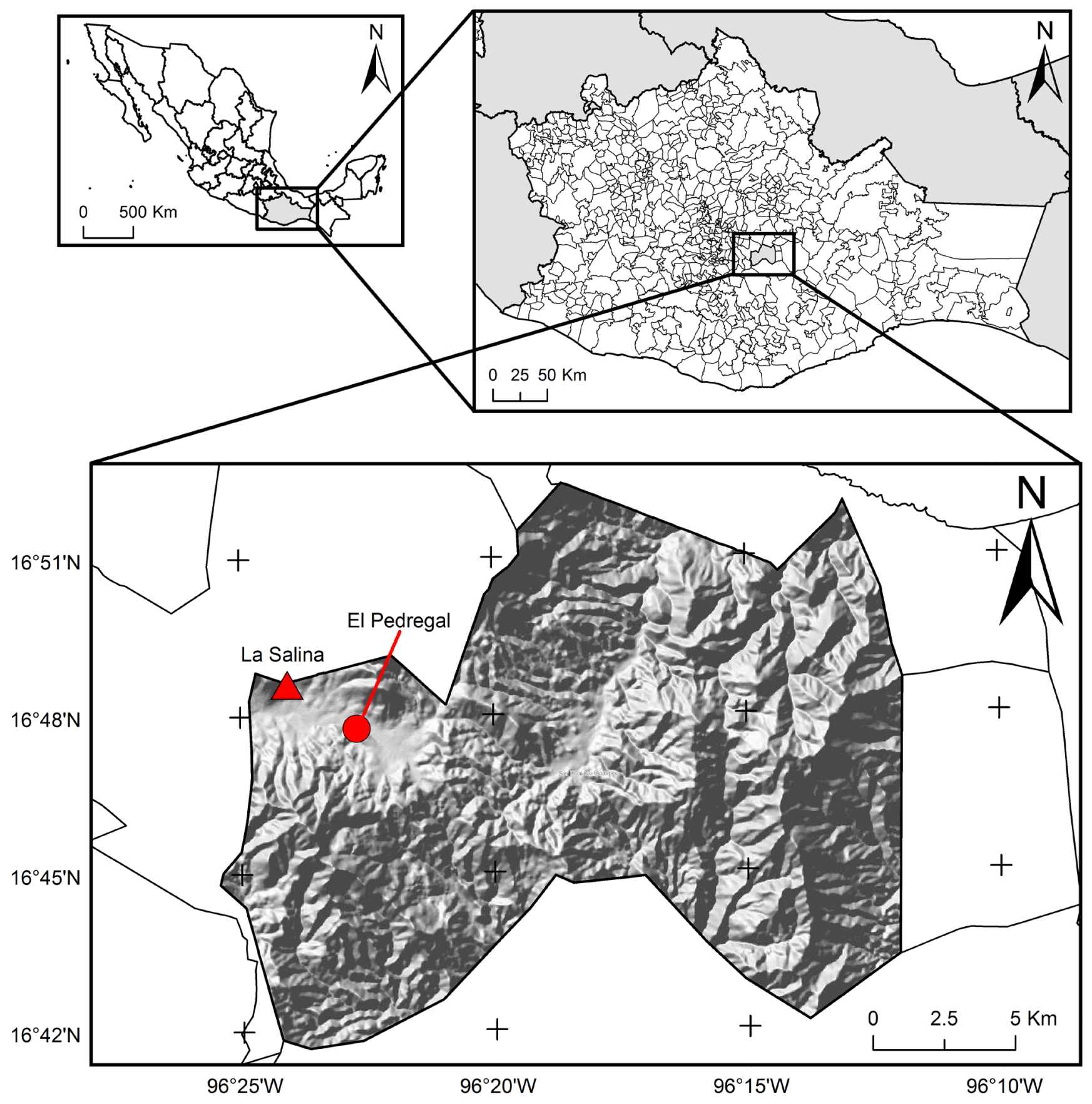

Figura 1 Ubicación geográfica y mapa hipsográfico de los yacimientos que forman la fauna local de San Dionisio Ocotepec. 
nama (Sánchez-Rojas et al., 2000).

La estratigrafia del sitio se compone principalmente por una alternancia de secuencias fluviales y volcanoclásticas (Figura 2). La columna tipo de la localidad se describe de base a cima en la Tabla 1.

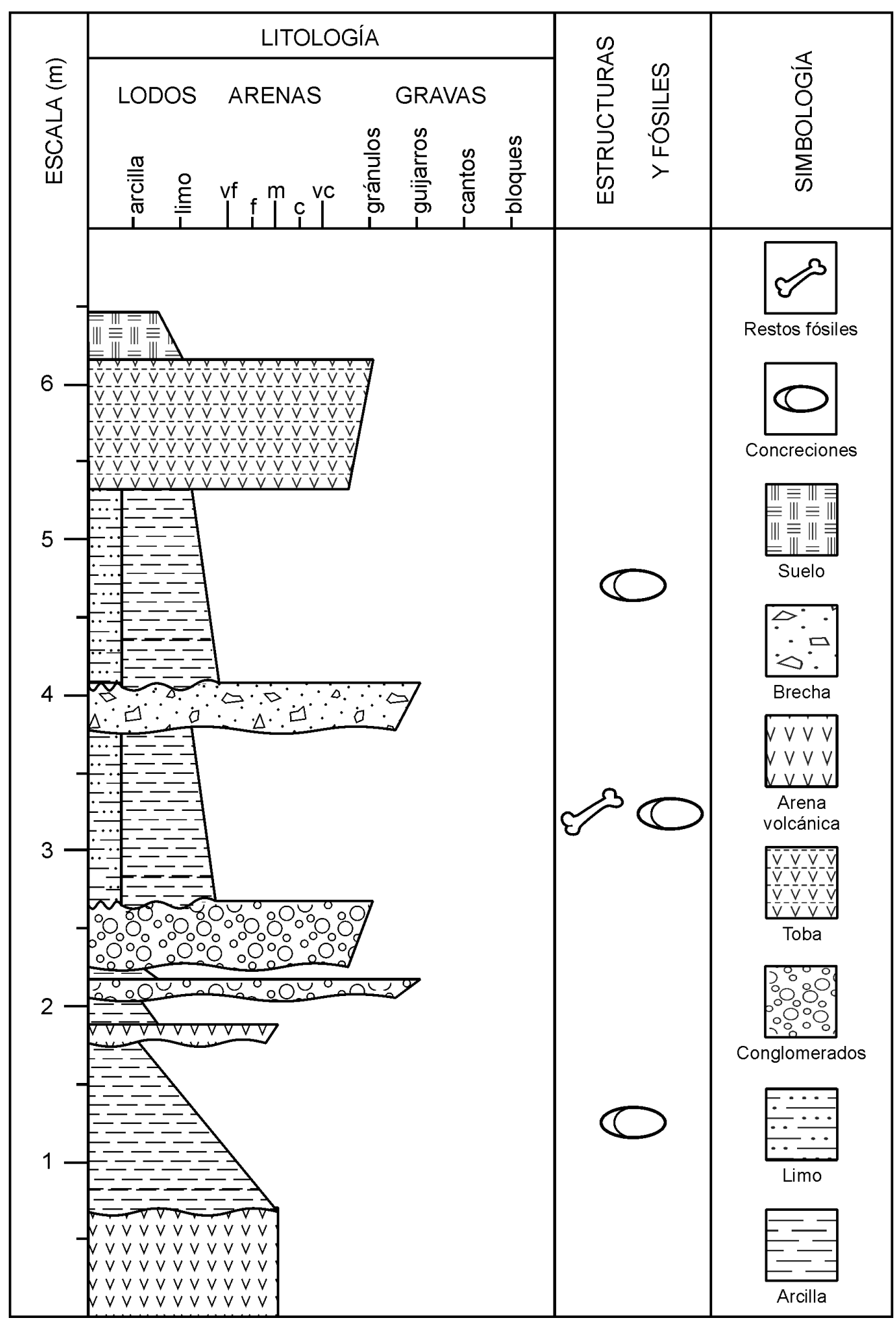

Figura 2 Columna estratigráfica tipo de la localidad. 
Tabla 1. Estratigrafía generalizada de San Dionisio Ocotepec, elaborada a partir de cuatro columnas litológicas.

\begin{tabular}{|c|c|}
\hline $\begin{array}{l}\text { Número de } \\
\text { estrato }\end{array}$ & Descripción \\
\hline 1 & $70 \mathrm{~cm}$ de arena volcánica andesítica fuertemente compactada. Contacto superior erosionado. \\
\hline 2 & $\begin{array}{l}109 \mathrm{~cm} \text { de arcilla con arena de color verde y con presencia de concreciones de carbonato de } \\
\text { calcio de hasta } 20 \mathrm{~cm} \text { de diámetro. Contacto superior erosionado. }\end{array}$ \\
\hline 3 & $\begin{array}{l}9 \mathrm{~cm} \text { de arena volcánica andesítica de color rojizo fuertemente consolidada, con lentes de } \\
\text { arcilla color café. Contacto superior neto. }\end{array}$ \\
\hline 4 & $20 \mathrm{~cm}$ de arcilla con limo de color café. Contacto superior erosionado. \\
\hline 5 & $\begin{array}{l}9 \mathrm{~cm} \text { de ortoconglomerados polimícticos con clastos de hasta } 2 \mathrm{~cm} \text { de diámetro. Contacto } \\
\text { superior neto, con presencia de oxidación. }\end{array}$ \\
\hline 6 & $10 \mathrm{~cm}$ de arcilla de color café. Contacto superior erosionado. \\
\hline $7-11$ & $\begin{array}{l}40 \mathrm{~cm} \text { de una intercalación de ortoconglomerados con brechas, ambos polimícticos y } \\
\text { compuestos de clastos de riolita, basalto, granito y feldespatos, con contactos netos entre } \\
\text { niveles y un contacto superior para el bloque de carácter fuertemente erosivo. }\end{array}$ \\
\hline 12 & $\begin{array}{l}112 \mathrm{~cm} \text { de arcilla con limo, de color café obscuro, con algunos clastos riolíticos y basálticos } \\
\text { embebidos en la matriz y presencia de carbonatación hacia la base. Presencia de restos fósiles } \\
\text { de vertebrados. Los fósiles se presentan tanto en la base como en la cima. Contacto superior } \\
\text { erosionado. }\end{array}$ \\
\hline 13 & $\begin{array}{l}28 \mathrm{~cm} \text { de brecha de color amarillento, con clastos de hasta } 10 \mathrm{~cm} \text { de diámetro. Contacto } \\
\text { superior erosivo. }\end{array}$ \\
\hline 14 & $\begin{array}{l}125 \mathrm{~cm} \text { de arcilla con limo de color negro, con lentes de arcilla rojiza con oxidación y } \\
\text { concreciones calcáreas de hasta } 10 \mathrm{~cm} \text { de diámetro máximo. Contacto superior neto, } \\
\text { ligeramente oxidado. }\end{array}$ \\
\hline 15 & $83 \mathrm{~cm}$ de toba retrabajada blanquecina con abundante oxidación. Contacto superior neto. \\
\hline 16 & $30 \mathrm{~cm}$ de suelo (regosol eútrico) residual moderno, con evidencia de pedogénesis in situ. \\
\hline
\end{tabular}

\section{Material y método}

Los fósiles se hallaron fortuitamente durante la construcción de un pozo de riego. En la zona del hallazgo se llevó a cabo el levantamiento de columnas litológicas para corroborar la ubicación estratigráfica de los restos fósiles. Adicionalmente, se llevaron a cabo salidas al campo para el levantamiento de la estratigrafía de la zona y la colecta de material fósil adicional en zonas aledañas al hallazgo original y en las barrancas y los arroyos de San Dionisio Ocotepec. El material colectado fue depositado en la Colección Paleontológica del Laboratorio de Paleontología de la Universidad
Michoacana de San Nicolás de Hidalgo (con las iniciales UM) y en la colección del proyecto de Museo de Sitio de San Dionisio Ocotepec (con las iniciales MSDO), el cual se encuentra bajo resguardo de la presidencia municipal.

Para la identificación de los restos fósiles se empleó bibliografía especializada y comparación directa. La comparación directa se llevó a cabo con los materiales de la Colección Científica del Laboratorio de Paleobiología, Instituto de Recursos, Universidad del Mar; la Colección Paleontológica del Laboratorio de Paleontología, Universidad Michoacana de San Nicolás de Hidalgo (UMSNH); la colección virtual del Museo de Paleontología de la Universidad de Michigan (UMMP VP); el material del Museo Estatal de Indiana (ISM) y del 
Museo Nacional de Historia Natural del Instituto Smithsoniano (USNM).

También se realizó un análisis de microdesgaste dental de baja magnificación a las piezas dentales de Mammuthus columbi (UM 1340, UM 1338), siguiendo el protocolo de Solounias y Semprebon (2002). En total, se llevaron a cabo tres moldes por pieza dental (maxilar y mandibular), de tres zonas: mesial, media y distal. Los moldes se observaron en un microscopio esteroscópico a 35x, del que se obtuvieron fotografias que fueron procesadas en el software Microware 4.02 (Ungar, 2002). Posteriormente, se obtuvo un promedio de hoyuelos y estrías para cada fotografia, que fue vertido en una base de datos de ungulados modelo modernos, tomada de Solounias y Semprebon (2002). Se hizo un análisis bivariante de promedio de estrías contra promedio de hoyuelos, así como análisis de funciones discriminantes canónicas (AFDC). Para el AFDG se usó un modelo de discriminación lineal y covarianza común, con las variables: promedio de hoyuelos y promedio de estrías; el soporte estadístico fue evaluado mediante una prueba de Lambda de Wilk, para corroborar la separación de categorías dietarias (pacedor, mixto y ramoneador). Finalmente, se llevó a cabo un análisis de mesodesgaste para proboscídeos, según la metodología propuesta por Saarinen et al. (2015); donde se promediaron los ángulos de los valles de dentina entre láminas de esmalte de seis zonas en los dos dientes reportados. Estos análisis se llevaron a cabo para inferir el tipo de hábitat en el que vivió la fauna de esta nueva localidad.

\section{Resultados}

En total se estudiaron más de 50 restos fósiles de los cuales, sólo 15 resultaron ser diagnósticos. La mayor parte de los restos procede del arroyo El Pedregal y sólo tres proceden de La Salina. Se reportan cuatro taxones en total: Mammuthus columbi, Equus cf. E. conversidens, Bison sp. y Bison latifrons.

\subsection{PALEONTOLOGÍA SISTEMÁTICA}

\author{
Orden Proboscidea Illigeri, 1811 \\ Familia Elephantidae Gray, 1821 \\ Mammuthus Brookes, 1828 \\ Mammuthus columbi (Falconer, 1858)
}

Material referido. UM 1340, M2 derecho completo, con fragmentos del maxilar; UM 1338, m2 derecho completo; UM 1339, vértebra lumbar (L1); UM 1341 unciforme derecho completo; UM 1342, costilla izquierda; MSDO 001, fragmento distal de defensa izquierda; MSDO 002, MSDO 003 (Figura 3) y MSDO 004, fragmentos de escápulas y MSDO 005, epífisis parcial de un húmero derecho.

Descripción y asignación taxonómica. Los molares (Figura 3 a-d) presentan la morfología típica de un elefántido elefantino, con dientes hipsodontes y loxodontes de láminas compactas (Agenbroad y Brunelle, 1992; Osborn, 1942). Las piezas presentan 6.5 placas por cada $100 \mathrm{~mm}$, lo que concuerda con el número diagnóstico para Mammuthus columbi de 6-7/100 mm en M2 y 5.5 - 6.5/100 mm en m2 (Osborn, 1942). La edad biológica del ejemplar corresponde con $26 \pm 1$ años, según el patrón de desgaste en las láminas que conforman los molares (Agenbroad y Brunelle, 1992).

La vértebra lumbar (Figura 3 e-g) presenta la morfología típica de un mamut, con procesos transversales delgados y no incorporados al centro vertebral, el cual posee una forma ovalada y presenta un margen inferior en forma de $\mathrm{U}$ (Hodgson et al., 2008; Olsen, 1972). Su comparación con el ejemplar UMMP VP 116967 , permitió identificarla como una lumbar 1 (L1), ya que en ambas el centro vertebral es más alto que ancho, la faceta articular de los procesos articulares craneales tienen forma de $\mathrm{V}$ en vista dorsal, los procesos transversos están orientados de forma caudal y son delgados, el margen dorsal de los procesos articulares caudales es redondeado y finalmente, la por- 
ción anterior del proceso espinoso inicia alrededor del $50 \%$ del ancho del centro vertebral.

Por su parte, en vistas ventral y posterior el unciforme (Figura $3 \mathrm{~h}$-i) presenta una forma subpiramidal, en vista anterior presenta una forma cuadrangular (Olsen, 1972); en vista medial, las superficies articulares del os magnum son anchas y se encuentran separadas por un istmo amplio hacia la zona volar; finalmente, la superficie articular inferior tiene un margen recto poco prominente (Hodgson et al., 2008).

Asumiendo que el dimorfismo sexual de defensas de mamut es similar al de elefántidos modernos, el fragmento de defensa (Figura 3 k-l) es referido como perteneciente a un macho, debido a la forma fuertemente triangular de la sección transversal de la misma, en contraposición con la sección oval de las hembras (Elder, 1970).

Al comparar la costilla de mamut asociada a los restos diagnósticos con costillas de Mammut americanum (ISM 71.3.261) y Mammuthus primigenius (USNM 23792), el elemento UM 1342 (Figura 3j) se asigna al género Mammuthus. La costilla de $M$. columbi referida es larga, delgada (Hodgson et al., 2008) y en sección transversal, presenta una marcada forma de $\mathrm{S}$, con prominentes cabeza y faceta articular caudal, un cuello relativamente largo, un tubérculo costal prominente y una tuberosidad íleo-costal con zonas de inserción muscular para el músculo íleo-costal torácico prominentes, además de un surco costal profundo. Es probable que la costilla proceda de la región media del cuerpo
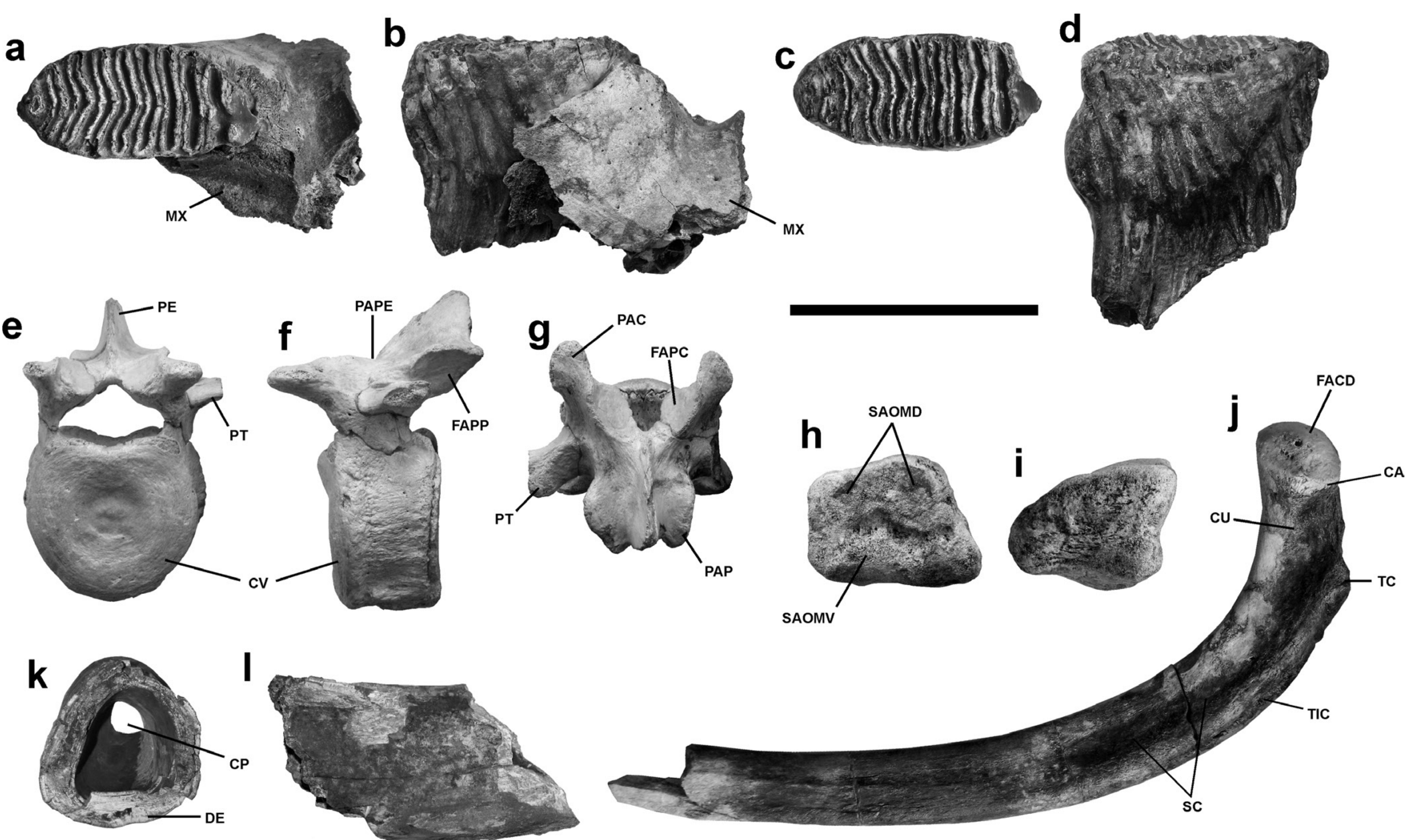

Figura 3 Restos fósiles de Mammuthus columbi. a, b) UM 1340 en vistas oclusal y labial; c, d) UM 1338 en vistas oclusal y labial; e, f, g) UM 1339 en vistas craneal, lateral izquierda y dorsal; h, i) UM 1341 en vistas medial y anterior; j) UM 1342 en vista especular posterior; k, I) MSDO 001 en vistas posterior y medial. Abreviaturas: MX, maxilar; PE, proceso espinoso; PT, proceso transverso; CV, centro vertebral; PAPE, porción anterior del proceso espinoso; FAPP, faceta articular de los procesos articulares caudales (posteriores); PAP, proceso articular caudal; PAC, proceso articular craneal; FAPC, faceta articular del proceso articular craneal; SAOMD, superficies articulares dorsales del os magnum; SAOMV, superficie articular ventral del os magnum; CP, cavidad pulpar; DE, dentina; FACD, faceta articular caudal; CA, cabeza; CU, cuello; TC, tubérculo costal; TIC, tuberosidad íleo-costal; SC, surco costal. Barra de escala: $10 \mathrm{~cm}$. 
por la curvatura del ángulo (Hodgson et al., 2008). El resto de las piezas se encontraron asociadas a las piezas diagnósticas, y presentan la morfología típica de Mammuthus (Olsen, 1972) por lo que se infiere que pertenecieron al mismo individuo.

\section{Orden Perissodactyla Owen, 1848 \\ Familia Equidae Gray, 1821 \\ Equus Linneo, 1758 \\ Equus cf. E. conversidens Owen, 1869}

Material referido. UM 1343, una vértebra cervical (C4-5) y UM 1344, una vértebra torácica de la región anterior (T5-7) (Figura 4).

Descripción y asignación taxonómica. La vértebra cervical (Figura 4) presenta las características típicas del género. Posee un centro vertebral inclinado en plano sagital anteroposteriormente, con forma acorazonada en las extremidades craneal y caudal; el proceso transverso sigue el eje del centro vertebral y se encuentra roto en su base; el proceso neural es corto y poco pronunciado, único en la región anterior y bifurcado en la posterior (formando una $\mathrm{W}$ ); posee una cresta ventral bien desarrollada hacia la región posterior y presenta forámenes laterales bien definidos (Barone, 1995; Klaus-Dieter et al., 2009; Pales y Garcia, 1981). Sólo se conserva el proceso articular caudal derecho, el cual rebasa el margen distal del centro vertebral en vista dorsal y posee una forma oval e inclinada medialmente.

La vértebra torácica (Figura 4) no posee el arco neural; presenta un centro vertebral inclinado en plano sagital anteroposteriormente y comprimido lateralmente en la región central; el centro posee forma semicircular, el margen dorsal es recto. Se reconoce como vértebra torácica por la posesión de las facetas costales craneal y caudal derechas y como séptima a quinta dorsal por la ausencia del foramen vascular, típico de vértebras torácicas posteriores (Barone, 1995; Klaus-Dieter et al., 2009). Se comparó la altura y la longitud del
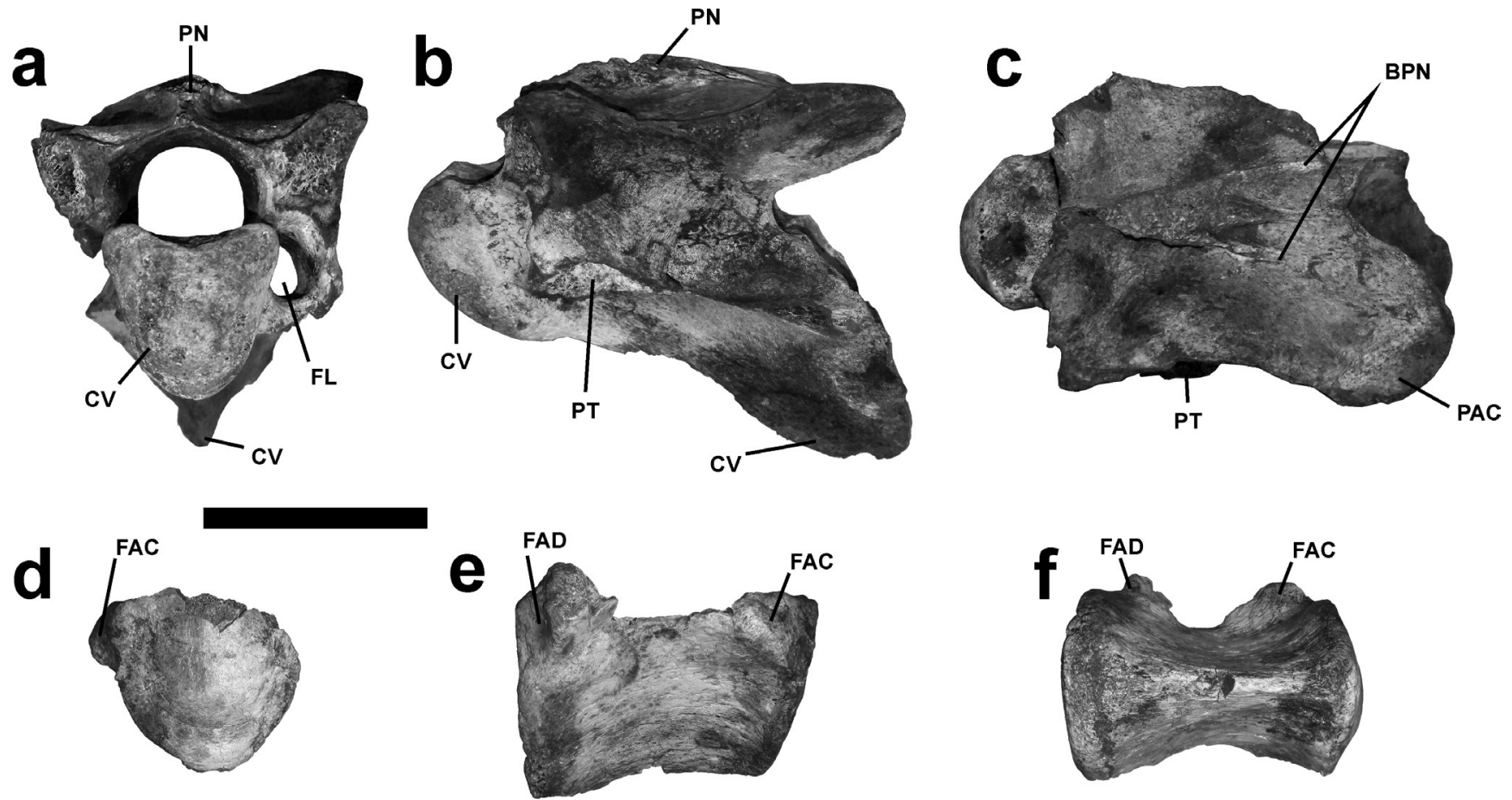

Figura 4 Restos fósiles de Equus cf. E. conversidens. a-c) UM 1343, en vistas craneal, lateral izquierda y dorsal. d-f) UM 1344, en vistas craneal, lateral derecha y ventral. Abreviaturas: PN, proceso neural; CV, centro vertebral; CV, cresta ventral; FL, foramen lateral; PT, proceso transversal; BPN, bifurcaciones del proceso espinoso; PAC, proceso articular caudal; FAC, faceta articular craneal; FAD, faceta articular caudal (distal). Barra de escala: $5 \mathrm{~cm}$. 
centro vertebral del elemento UM 1344 con Equus conversidens y Equus cf. E. cedralensis de la Colección Paleontológica de la UMSNH y el elemento UM 1344 es similar en dimensiones a E. conversidens (Tabla 2).

Debido a la similitud de talla entre los elementos UM 1343 y UM 1344 y debido a que Equus conversidens es el équido de talla media reportado para el Rancholabreano de México (Priego-Vargas et al., 2016), ambos elementos se asignan a este taxón.

\section{Orden Artiodactyla Owen, 1848 \\ Familia Bovidae Gray, 1821 \\ Bison (Hamilton-Smith, 1827) Bison sp.}

\begin{abstract}
Material referido. UM 1345, un acetábulo derecho.

Descripción y asignación taxonómica. El acetábulo (Figura 5) muestra la morfología típica de un bovino de gran talla, que se corresponde con Bison (McCuaig-Balkwill y Cumbaa, 1992). Posee un borde acetabular semicircular, la superficie semilunar es amplia y se encuentra casi cerrada, con una incisura acetabular estrecha y una fosa acetabular profunda y notoria. La superficie semilunar del pubis emerge de una eminencia íleo-púbica notoria y amplia; en vista cenital, el borde aceta-
\end{abstract}

bular se encuentra hendido en su porción dorsal, lo que permite apreciar parte de la superficie semilunar inferior (Barone, 1995; Schmid, 1972).

Bison latifrons (Harlan, 1825)

Material referido. UM 1346, parte proximal del radio derecho de un macho adulto, preservado desde el sulco tendíneo y hasta la zona articular proximal y con la cabeza completa (Figura 6).

Descripción y asignación taxonómica. El radio presenta las características típicas de un bovino de gran talla y se identifica como Bison por su morfología general (McCuaig-Balkwill y Cumbaa, 1992). Por debajo de la repisa de la tuberosidad radial y hacia la zona medial, se presenta una cicatriz para el músculo bíceps braquial bien desarrollada. Ésta posee forma cuadrada y se extiende hacia la zona posterior. La repisa de la tuberosidad radial es amplia y se encuentra expandida medialmente. La tuberosidad lateral o externa es amplia y posee un relieve lateral de inserción en un ángulo aproximado de $45^{\circ}$ con respecto al eje articular. Este relieve es amplio y la zona de inserción del extensor dorsal del dedo (extensor digiti quarti propius) es mayor y dispuesta de forma más anterior que la zona de inserción del extensor lateral del dedo (digitorum communis). El proceso coronoide es redondeado y poco prominente; la hendidura central es

Tabla 2. Medidas de vértebras torácicas seleccionadas de Equus de México. Abreviaturas: LCDe, longitud máxima del cuerpo vertebral, incluyendo el diente; BFcr, ancho máximo en la facies articularis cranialis; BFcd, ancho máximo en la facies articularis caudalis. Medidas en $\mathrm{mm}$.

\begin{tabular}{|l|l|c|c|c|c|c|}
\hline Elemento & Taxón & LCDe & BFcr & BFed & $\begin{array}{c}\text { Altura del } \\
\text { centro }\end{array}$ & $\begin{array}{c}\text { Largo del } \\
\text { centro }\end{array}$ \\
\hline UM 1344 & Equus cf. E. conversidens & 64.9 & 48.2 & 51.2 & 48.1 & 70.6 \\
\hline UM 1346 & E. cedralensis & 33.6 & 32.1 & 35.1 & 27.5 & 39.9 \\
\hline UM 1347 & E. cedralensis & 37.8 & 28.8 & 31 & 25.2 & 36.3 \\
\hline UM 1348 & E. cedralensis & 40.9 & 30.6 & 32.5 & 29.6 & 44.4 \\
\hline UM 1349 & E. cedralensis & 40.9 & 33.8 & 32.2 & 28.7 & 40.6 \\
\hline UM 1350 & E. cedralensis & 40.1 & 29.8 & 29.2 & 26.8 & 42.3 \\
\hline UM 1351 & E. conversidens & 63.9 & 37.5 & 45.6 & 43.3 & 68.4 \\
\hline UM 1352 & E. conversidens & 58.2 & 45.3 & 49.2 & 50.8 & 68.2 \\
\hline UM 1353 & E. conversidens & 51.6 & 42.3 & 47.4 & 48.8 & 61.8 \\
\hline
\end{tabular}



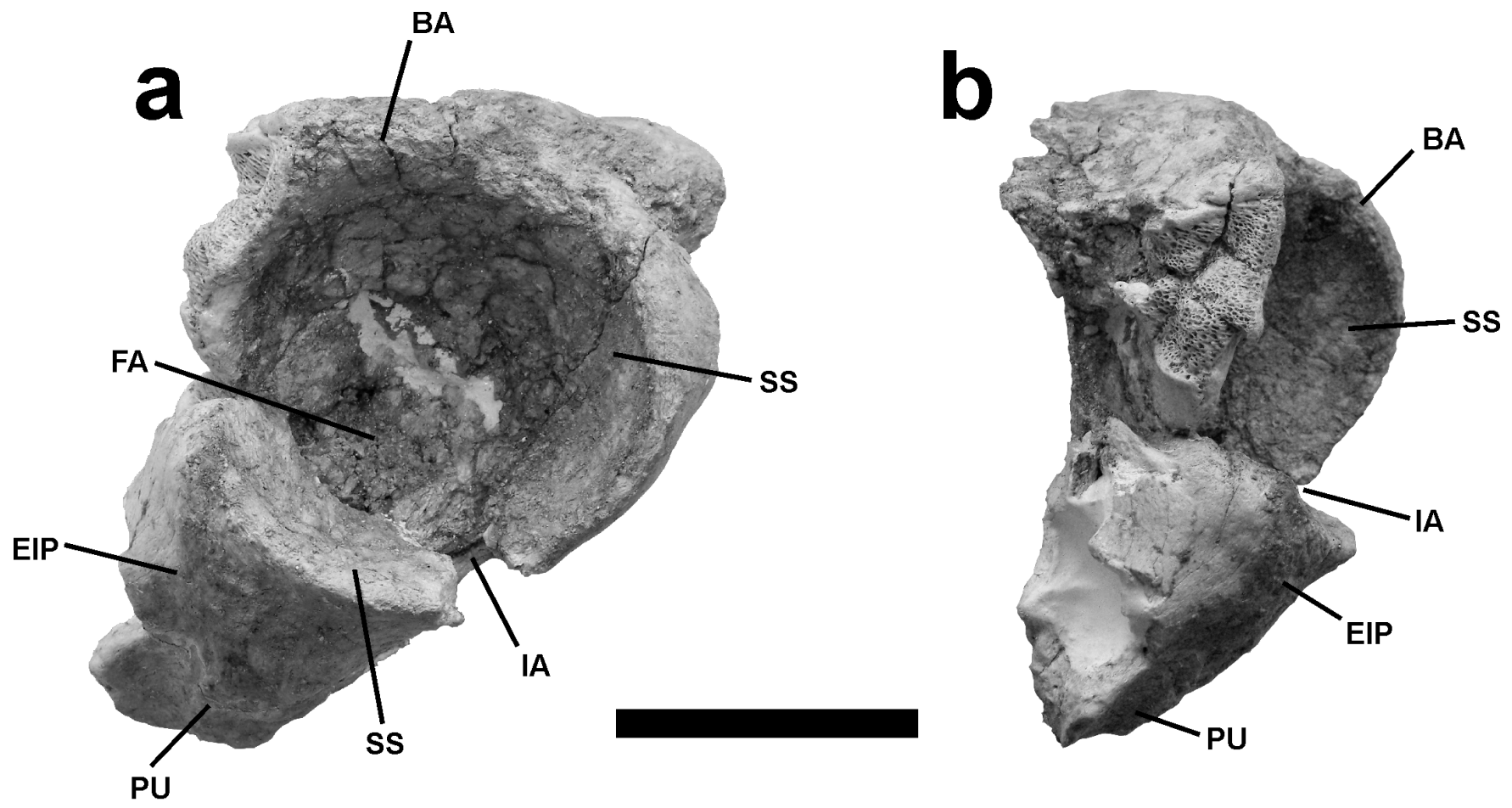

Figura 5 Acetábulo de Bison sp. UM 1345. a) Vista lateral, b) vista caudal. Abreviaturas: BA, borde acetabular; SS, superficie semilunar; IA, incisura acetabular; FA, fosa acetabular; EIP, eminencia íleo-púbica; PU, pubis. Barra de escala: $5 \mathrm{~cm}$.

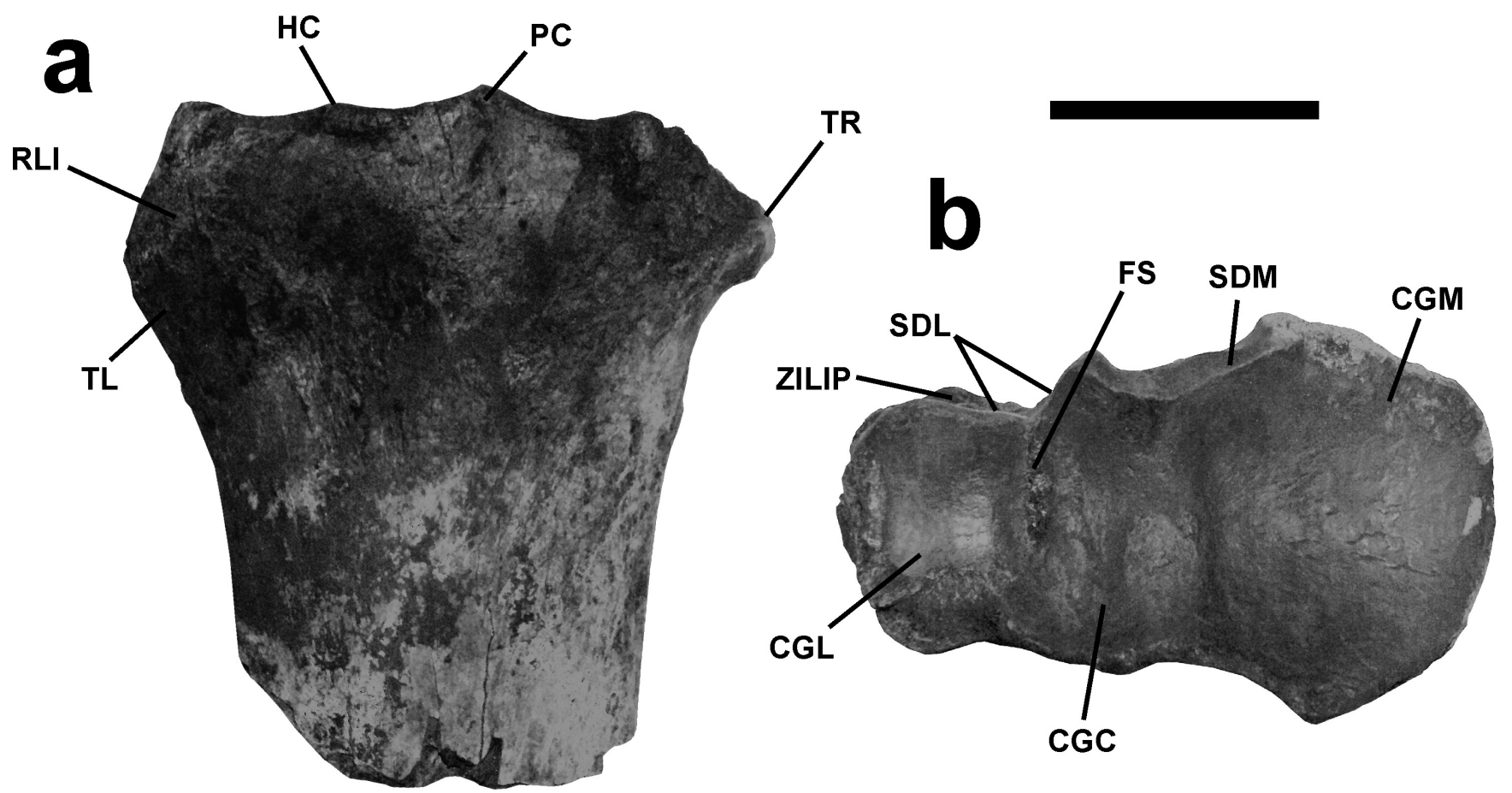

Figura 6 Radio de Bison latifrons, UM 1346. a) Vista anterior, b) vista dorsal. Abreviaturas: RLI, relieve lateral de inserción; TL, tuberosidad lateral; HC, hendidura central; PC, proceso coronoides; TR, repisa de la tuberosidad radial; ZILIP, zona de inserción del ligamento interóseo proximal; SDL, superficies diartrodiales laterales; SDM, superficie diartrodial medial; FS, foseta sinovial; CGM, cavidad glenoidea medial; CGC, cavidad glenoidea medial (central); CGL, cavidad glenoidea lateral. Barra de escala: $5 \mathrm{~cm}$. 
amplia y presenta una curva suave; en vista cenital, la faceta articular proximal presenta una cavidad glenoidea medial (o fosa capitular) amplia y separada de la cavidad glenoidea media y lateral, que son más pequeñas y cuadrangulares. Finalmente, la hendidura articular de la ulna es amplia y presenta un ángulo obtuso de aproximadamente $135^{\circ}$ (Olsen, 1960; McCuaig-Balkwill y Cumbaa, 1992; Barone, 1995; Pales y Garcia, 1981; France, 2009). Para el Rancholabreano de Norteamérica y México se reportan cuatro especies de bisontes (McDonald, 1981; Ferrusquía-Villafranca et al., 2010), de los cuales dos eran gigantes: el eurasiático Bison alaskensis y el autóctono Bison latifrons. Sin embargo, consideramos que este radio perteneció a $B$. latifrons por sus rasgos morfológicos. Comparamos la morfología de radios descrita para $B$. priscus debido a que $B$. alaskensis es conocido únicamente de cráneos y algunos autores lo refieren como una subespecie de B. priscus (e.g. Guthrie, 1966), además de que es parte del grupo de bisontes de origen eurasiático (McDonald, 1981).

Los radios de $B$. priscus son largos y gráciles, pero con epífisis gruesas, lo que les da un aspecto de tener una cabeza amplia (Shpansky et al., 2016), en contraposición, el elemento UM 1346 no presenta una región epifisiaria notablemente expandida (Figura 6). En B. priscus la ulna se encuentra fuertemente anquilosada al radio (Prat et al., 2010; Shpansky et al., 2016), mientras que en UM 1346, se observan claramente la superficie de sinostosis radio-ulnar y la zona de inserción del ligamento interóseo proximal y no existe evidencia de anquilosis, lo que sugiere la presencia de un espacio interóseo proximal bien desarrollado. Además, en B. priscus (Figura 7) el relieve lateral de inserción se presenta en un ángulo cercano a $90^{\circ}$, presenta una tuberosidad lateral muy grande, alta y ligeramente hendida, con un borde dorsal que se encuentra a la misma altura del proceso coronoide y en algunos ejemplares, incluso lo excede en altura (Prat et al., 2010). Ninguna de estas características está presente en UM 1346.

En Oaxaca, se ha reportado la presencia de Bison antiquus (Jiménez-Hidalgo et al., 2011, 2013). El es- pécimen UM 1346 se asigna a un ejemplar macho de $B$. latifrons por su talla (Figura 6) y por comparación con radios de B. latifrons (UM 1264) y B. antiquus (UM 1347 y UMPE 0004). B. antiquus presenta una foseta sinovial en forma de moño, que ingresa a las cavidades glenoideas (medial y media) desde el punto intermedio de las superficies diartrodiales, mientras que la foseta de $B$. latifrons es recta, está dirigida hacia la parte anterior e ingresa únicamente a la cavidad glenoidea media desde el punto central de las superficies diartrodiales laterales. En B. antiquus el margen anterior de la cavidad glenoidea medial es ligeramente subtriangular, mientras que en $B$. latifrons forma un ángulo bastante prominente. En $B$. latifrons la repisa de la tuberosidad radial está más acentuada y el proceso coronoide es más redondeado. En la región posterior, la superficie diartrodial medial de $B$. latifrons es más grande y larga, rebasando el
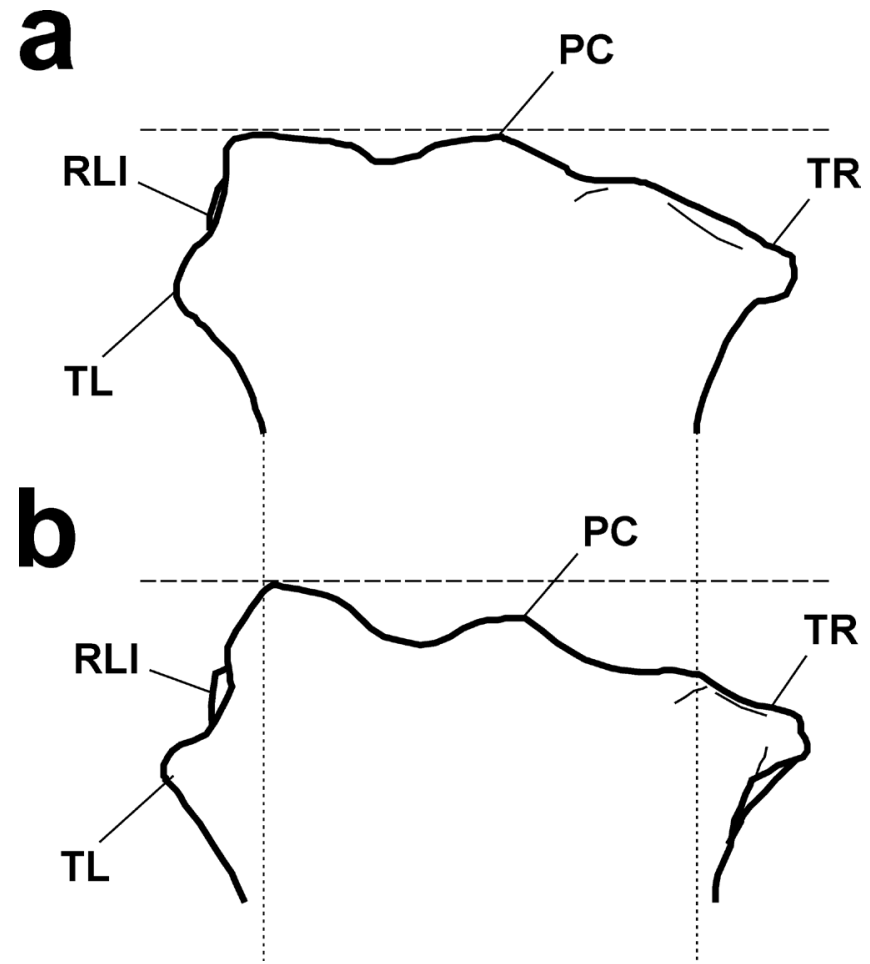

Figura 7 Comparación de la región proximal en radios de a) Bison latifrons y b) B. priscus en vista anterior. La línea punteada gruesa representa el punto más alto del tubérculo lateral. La línea punteada delgada indica el ancho relativo de la diáfisis en B. Iatifrons. Abreviaturas igual que en la figura 6. Los diagramas no están a escala. 
margen ventral de las superficies diartrodiales laterales. Además, la superficie diartrodial medial de $B$. latifrons es menos pronunciada que en $B$. antiquus. Adicionalmente, la inserción del ligamento interóseo proximal es mucho más amplia y expandida posteriormente en $B$. antiquus. Finalmente, el ancho y largo de las superficies articulares proximales del radio UM 1346 se corresponden en sus dimensiones con las reportadas de $B$. latifrons machos (Figura 8).

\subsection{INFERENGIA ALIMENTARIA}

El único taxón analizado fue Mammuthus columbi, dado que fue el único con piezas dentales conser- vadas. El análisis de microdesgaste dental (Figura 9) sugiere que, en los últimos días de su vida, el individuo de mamut fue pacedor. Esta asignación dietaria se soporta con el análisis de funciones discriminantes, donde la pieza mandibular se asigna a dicho gremio con una probabilidad de $61 \%$ y la maxilar con una probabilidad de $45.3 \%$. Además, la prueba de Lambda de Wilk muestra diferencias estadísticamente significativas entre gremios (ramoneadores, mixtos, pacedores), reforzando así esta asignación.

A diferencia del análisis de microdesgaste dental, el análisis de mesodesgaste representa la preferencia dietaria a lo largo de la vida de un individuo (For-

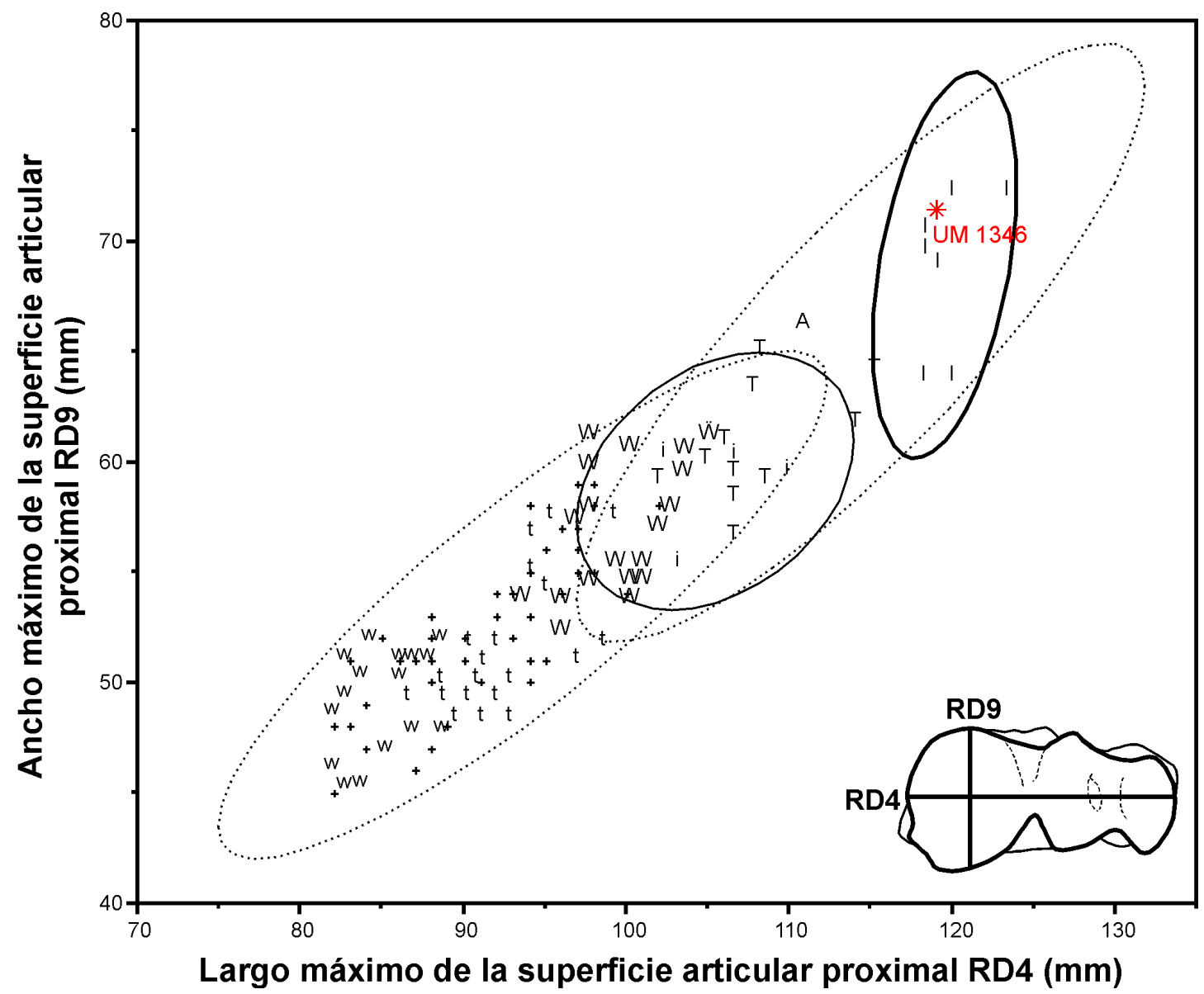

Figura 8 Análisis bivariante de radios de bisontes. Las elipses representan el $95 \%$ de la confianza. Elipse punteada inferior de $B$. antiquus, elipse punteada superior de $B$. latifrons. Elipse sólida delgada de hembras de $B$. latifrons. Elipse sólida gruesa de machos de $B$. latifrons. Simbología i, I: hembras y machos (respectivamente) de Reserva de American Falls, Idaho; $t$, T: hembras y machos (respectivamente) de Lipscomb, Texas; w, W: hembras y machos (respectivamente) de Horner II, Wyoming; A: macho de Gallelli Gravel Pit, Alberta; +: Horner II, Wyoming (datos sin sexo especificado). I, i: Bison laifrons. T, t, W, w, A, +: Bison antiquus. Datos tomados de (Stevens, 1978; Todd, 1987; Todd et al., 1992; Wilson et al., 2008). 


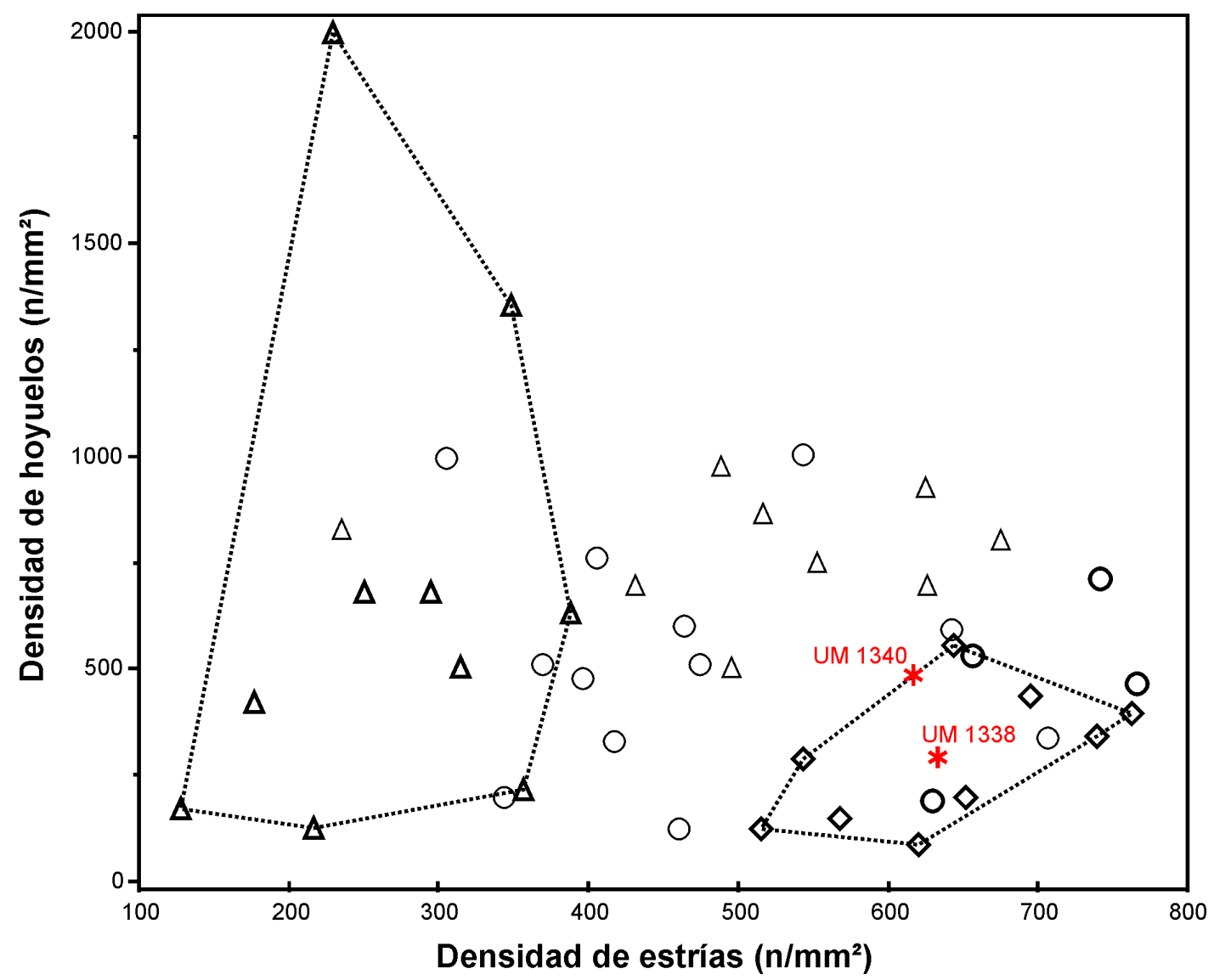

Figura 9 Análisis bivariante de microdesgaste dental de baja magnificación (35x), datos de Solounias y Semprebon (2002). Simbología: triángulos con línea gruesa: ramoneadores, triángulos con línea delgada: frugívoros, círculos con líneas delgadas: mixtos estacionales, círculos con líneas gruesas: mixtos no estacionales, rombos:pacedores. Ecoespacios principales delimitados por polígonos punteados.

telius y Solounias, 2000; Saarinen et al., 2015). Los fósiles analizados poseen un promedio de ángulos de $125.7^{\circ}$ (m2) y de $125.5^{\circ}$ (M2). Estos valores (Figura 10) sugieren que el ejemplar de $M$. columbi de este estudio tenía una dieta dominada por plantas C4 $(>70 \%)$, lo cual es consistente con un hábito pacedor no estricto (Saarinen et al., 2015).

\section{Discusión}

\subsection{ORIGEN DE BISON LATIFRONS Y EDAD RELATIVA DE SAN DIONISIO OGOTEPEG}

Se considera que el Rancholabreano inició hace $160 \mathrm{Ka}$ antes del presente, usando como taxón índice al género Bison y en particular a B. latifrons (Bell et al., 2004). Sin embargo, los registros tem- pranos usados para establecer esta edad son dudosos (Scott y Lindvall, 1970; Scott et al., 1982; Haynes 1985). Por otro lado, la evidencia molecular apunta a que los bisontes invadieron América del Norte en dos oleadas, la más temprana situada entre 195 y $135 \mathrm{Ka}$, pero representada únicamente en Beringia por formas eurasiáticas como Bison priscus (Froese et al., 2017). Los autóctonos americanos como Bison latifrons aparecieron posteriormente en América continental (McDonald, 1981). Esta especie se conoce de un intervalo de entre $120 \mathrm{Ka}$ (Froese et al., 2017; Miller et al., 2014) y 6.4 a 7.7 $\mathrm{Ka}$ antes del presente (Dillehay, 1974; Gagliano, 1967). Por consiguiente, asignamos la fauna local de San Dionisio Ocotepec a la edad de mamíferos terrestres norteamericanos del Rancholabreano (Savage, 1951; Bell et al., 2004). 


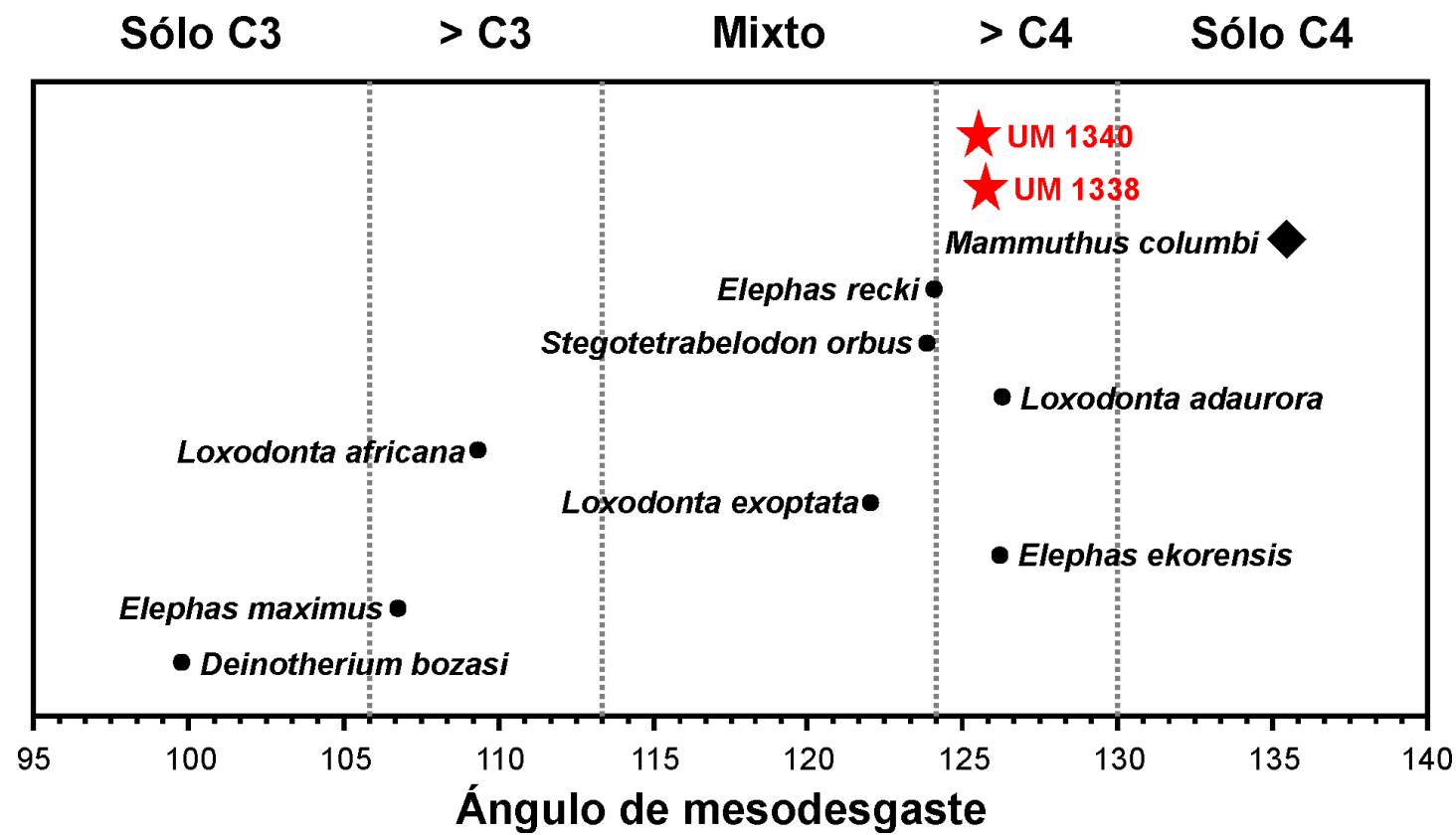

Figura 10 Mesodesgaste de proboscídeos y su relación con valores isotópicos de carbono y gremios conocidos. Modificado de Saarinen et al. (2015).

\subsection{DISTRIBUGIÓN GEOGRÁFIGA}

Mammuthus columbi se ha reportado en casi todos los estados del país, con excepción de Quintana Roo, Yucatán, Campeche, Tabasco, y Colima (Arroyo-Cabrales et al., 2007). Para Oaxaca se tienen al menos seis reportes de la especie (Arroyo-Cabrales et al., 2003; Pérez-Crespo et al., 2008), de los que dos registros (Guadalupe Victoria y San Pablo Etla) son referibles a la región noroeste de los Valles Centrales (Pérez-Crespo et al., 2008). El registro de este trabajo extiende la distribución de la especie hacia el sur en aproximadamente 55 $\mathrm{Km}$.

Por otra parte, el género Equus se ha registrado en casi todas las provincias morfotectónicas del país, con excepción de la Sierra Madre Occidental y la Planicie Costera del Golfo-Sur (Priego-Vargas et al., 2016). Equus conversidens se presenta en las provincias morfotectónicas de: Sierras y Planicies del Noroeste, Cordilleras y Planicies de Chihuahua-Coahuila, Meseta Central, Sierra Madre Oriental, Cinturón Volcánico Transmexicano, Planicie Costera del Golfo-Norte, Península de Yucatán, Sierra Madre de Chiapas y Sierra Madre del Sur (Priego-Vargas et al., 2016). En Oaxaca se ha reportado previamente la presencia de $E$. excelsus en Ejutla y Tehuantepec (Pérez-Crespo et al., 2008), E. mexicanus en Teposcolula (Pérez-Crespo et al., 2008) y la Mixteca Alta (Jiménez-Hidalgo et al., 2012). Finalmente, E. conversidens ha sido reportado únicamente para la Mixteca (Jiménez-Hidalgo et al., 2012). El posible registro de E. conversidens de San Dionisio Ocotepec conecta las localidades de la Mixteca Alta con las de la depresión central de Chiapas (Carbot-Chanona y Gómez-Pérez, 2014). Los bisontes (Bison spp.) están más o menos bien representados en México, sus registros son abundantes en las provincias morfotectónicas de Meseta Central, Sierra Madre Oriental y Cinturón Volcánico Transmexicano; mientras que hay pocos registros en las provincias de Península de Baja California, Sierras y Planicies del Noroeste, Cordilleras y Planicies de Chihuahua-Coahuila y Península de Yucatán (Ferrusquía-Villafranca et al., 2010). Por otro lado, se presenta sólo un registro de Bison tanto en la provincia de la Sierra Madre del Sur (Jiménez-Hidalgo et al., 2011, 2013) como en la Sierra Madre de Chiapas (Carbot-Chanona y Vázquez-Bautista, 2006). En Oaxaca, este registro 
corresponde con la fauna local de Viko Vijin en la Mixteca alta, donde se reporta la presencia de la especie más abundante en México, Bison antiquus (Jiménez-Hidalgo et al., 2013).

\subsection{ESTRATIGRAFÍA}

El análisis estratigráfico sugiere que los depósitos de San Dionisio son de carácter predominantemente fluvial. La presencia de concreciones de carbonato de calcio en forma de caliche y carbonatación sugiere la presencia de condiciones áridas (Schlesinger, 1982). Estas condiciones se encuentran en facies someras dominadas por el depósito de arcillas finas (en los estratos 2, 12 y 14), que sobreyacen sedimentos alóctonos de origen volcánico y perturbaciones estocásticas del régimen fluvial evidenciadas por la presencia de conglomerados y brechas. Estas condiciones sugieren que las facies someras presentaban pedogénesis in situ incompleta, que no dio origen a paleosuelos, pues este proceso se vio interrumpido en varias ocasiones por el depósito de nuevos sedimentos, culminando hacia la cima por el arrastre de sedimentos volcánicos alóctonos y la formación de suelos modernos.

\subsection{TAFONOMÍA}

Los restos fósiles de San Dionisio Ocotepec sugieren que el efecto de ponderación temporal (Time-averaging) es mínimo, particularmente en el sitio "El Pedregal", donde se encuentran los taxones: Mammuthus columbi, Equus cf. E. conversidens y Bison latifrons. Estos restos fósiles presentan escasas evidencias de intemperismo: poseen una superficie ósea con poco agrietamiento, no se presenta descamación y las superficies articulares presentan grietas en mosaico; lo que sugiere que los restos tuvieron un tiempo de exposición al medio que va de los días a los tres años como máximo (Behrensmeyer, 1978). Adicionalmente, la evidencia muestra que el arrastre de las piezas fue escaso, los bordes son completos y hay presencia de fracturas en espiral (causadas por compresión) con márgenes angulosos (Fernández-Jalvo y Andrews,
2003; 2016). El radio de Bison latifrons se encuentra dentro del grupo II de Voorhies, lo que indica que su transporte fue moderado, principalmente como carga tractiva (Voorhies, 1969). Finalmente, los restos de $M$. columbi descritos tienen índices de transporte fluvial de entre $53.9 \%$ y $86.8 \%$ (Frison y Todd, 1986), estos elementos corresponden a un único individuo adulto y fueron encontrados asociados. Sin embargo, el resto del esqueleto no se pudo rescatar debido a la construcción de un pozo de riego.

\subsection{DESGASTE DENTAL}

El microdesgaste dental representa la preferencia dietaria con un margen de horas a días antes de la muerte (Solounias y Semprebon, 2002). Estos patrones reflejan el tipo de vegetación disponible cercana a la zona de depósito (Solounias y Moelleken, 1994). Por otro lado, el mesodesgaste refleja la preferencia dietaria a lo largo de la vida útil de la pieza dental analizada (Saarinen et al., 2015). Estos patrones de desgaste se emplean para llevar a cabo inferencias paleoambientales y predicciones dietarias para las especies con muestras de al menos diez individuos (Fortelius y Solounias, 2000). Estas aproximaciones ofrecen información ambiental limitada con tamaños de muestra inferiores a 10, incluyendo en ocasiones un individuo (Bernor et al., 2014; Danowitz et al., 2016; Hayek et al., 1992). Sin embargo, estas inferencias deben ser tomadas como tentativas y no ser empleadas para hacer generalizaciones paleoecológicas de las especies o del sitio de depósito.

Los resultados de los análisis de desgaste en el ejemplar de $M$. columbi indican que el individuo fue un pacedor no estricto que se alimentó preferentemente de pastos en los últimos días a horas antes de su muerte. Esto se corresponde con lo que muestran otros estudios de microdesgaste llevados a cabo con esta especie en otras regiones de México (Gutiérrez Bedolla et al., 2016). En el caso del mesodesgaste, el promedio de ángulos de los molares de M. columbi de este estudio fue de $125.6^{\circ}$, lo que sugiere una dieta mixta, dominada por plantas C4 (poáceas). Esto es distinto a lo encontrado para 
la misma especie en Rancho la Brea (Saarinen et al., 2015), con un promedio de ángulos de $135.5^{\circ}$ y a lo encontrado en ejemplares del centro-este de Puebla, donde se obtuvo un promedio de $135^{\circ}$ (Carbot-Chanona et al., 2018), lo que sugiere que en estos sitios $M$. columbi era un pacedor casi estricto, con un consumo de plantas C4 de más de $90 \%$ (Saarinen et al., 2015). A pesar de sugerir la presencia de pastizales, la información del desgaste no es suficiente, por lo que se requieren de estudios adicionales, tales como mineralógicos y palinológicos.

\subsection{PALEOAMBIENTE}

Actualmente el relieve de la zona es irregular, con elevaciones pronunciadas hacia el sur (donde inicia la Sierra Madre del Sur) y hacia el norte, planicies que se conectan a la región fisiográfica de valles y lomeríos del centro del estado. Esta topografía debió de haber sido similar durante el Pleistoceno tardío, pues los afloramientos aledaños pertenecen a formaciones que datan del Cretácico tardío ( $c a$. $91-71.5 \mathrm{Ma}$ ) y existen pocos indicios de depósitos posteriores más recientes.

La ocurrencia conjunta de M. columbi y Equus cf. E. conversidens también sugiere la presencia de zonas abiertas con pastizales en las partes planas, como lo indican diversos estudios con estos taxones en México (Bravo-Cuevas et al., 2011; Gutiérrez Bedolla et al., 2016; Marín-Leyva et al., 2016). Aparentemente esto entra en contraposición con el hábitat forestal sugerido para Bison latifrons (McDonald, 1981). Sin embargo, no existe evidencia que sugiera que efectivamente, este era el ambiente preferido de este bóvido y pudo habitar en otros tipos de ecosistema, como el inferido para San Dionisio Ocotepec.

\section{Conclusiones}

Los sedimentos de San Dionisio Ocotepec datan del Pleistoceno tardío, específicamente a la NALMA del Rancholabreano y consta de al menos tres taxones a nivel específico: Mammuthus columbi, Equus cf. E. conversidens y Bison latifrons. La presencia del mamut columbino y del onagro mexicano durante el Rancholabreano en México es relativamente común. La presencia de estas especies da conectividad a localidades de la región noroeste de Oaxaca con aquellas de Chiapas. Por otra parte, en este estudio corroboramos la presencia de Bison latifrons en los Valles Centrales del estado de Oaxaca, lo que marca la distribución más austral de la especie en el continente, extendiendo en más de $447 \mathrm{~km}$ su rango de distribución geográfica conocida.

La información estratigráfica sugiere que el ambiente sedimentario predominante en San Dionisio fue fluvial, con aporte principal de terrígenos y de forma secundaria, tobas, brechas y conglomerados. Considerando la evidencia de la topografía, la estratigrafía y de los restos fósiles, sugerimos que las partes planas de San Dionisio Ocotepec fueron ocupadas por pastizales.

\section{Agradecimientos}

Agradecemos a las autoridades y a los pobladores del municipio de San Dionisio Ocotepec por las facilidades brindadas para la consecución de este trabajo, especialmente al señor Ceferino López por su apoyo durante las salidas a campo y a su gestión dentro del municipio. Agradecemos también a Rosalía Guerrero Arenas y a Nicolás Roberto Chimento por sus valiosos comentarios, que ayudaron al mejoramiento del manuscrito.

\section{Referencias}

Agenbroad, L.D., Brunelle, A.R., 1992, Analysis of Mammoth Dentition (How to be a tooth fairy for elephants). Teacher \& Classroom Kit and Manual (Grades 4-12). Northern Arizona University and the Mammoth Site of Hot Springs, South Dakota.

Arroyo-Cabrales, J., Carreño, A.L., LozanoGarcía, S., Montellano-Ballesteros, 
M., Cevallos-Ferriz, S., Corona, E., Espinoza-Arrubarrena, L., Guzmán, A.F., Magallón-Puebla, S., Morán-Zenteno, D.J., Naranjo-García, E., Olivera, M.T., Polaco, O.J., Sosa, S.N., Téllez-Duarte, M., TovarLiceaga, R.E., Vázquez-Selem, L., 2008, La diversidad en el pasado, en Sarukhán, J.K., Soberón, J. (eds.), Capital Natural de México, Vol. 1: Conocimiento Actual de La Biodiversidad. CONABIO, México, D. F., 227-262.

Arroyo-Cabrales, J., Polaco, O.J., Johnson, E., 2002, La mastofauna del Cuaternario tardío en México, en Montellano-Ballesteros, M., Arroyo-Cabrales, J. (eds.), Avances En Los Estudios Paleomastozoológicos En México, Instituto Nacional de Antropología e Historia, Colección Científica, México, D. F., 103-123.

Arroyo-Cabrales, J., Polaco, O.J., Johnson, E., Guzmán, A.F., 2003, The distribution of the genus Mammuthus in Mexico: Denisea, 9(1), 27-39.

Arroyo-Cabrales, J., Polaco, O.J., Laurito, C., Johnson, E., Alberdi, M.T., ValerioZamora, A.L., 2007, The proboscideans (Mammalia) from Mesoamerica: Quaternary International, 169-170, Special Issue, 17-23.

Barone, R., 1995, Anatomia comparata dei mammiferi domestici. Vol. 1 Osteologia, Edagricole, Edizioni Agricole, Bologna, Italia, 690 p.

Behrensmeyer, A.K., 1978, Taphonomic and ecologic information from bone weathering: Paleobiology, 4, 150-162.

Bell, G.J., Lundelius Jr., E.L., Barnosky, A.D., Graham, R.W., Lindsay, E.H., RuezJr., D.R., Semken Jr., H.A., Webb, S.D., Zakrzewski, R.J., 2004, The Blancan, Irvingtonian, and Rancholabrean Mammal Ages, en Woodbourne, M.O. (ed.), Late Cretaceous and Cenozoic Mammals of North America. Biostratigraphy and Geochronology, Columbia University Press, New York,
232-314.

Bernor, R.L., Semprebon, G.M., Damuth,J., 2014,

Maragheh ungulate mesowear: interpreting paleodiet and paleoecology from a diverse fauna with restricted sample sizes: Annales Zoologici Fennici, 51, 201-208.

Bravo-Guevas, V.M., Jiménez-Hidalgo, E., Priego-Vargas, J., 2011, Taxonomía y hábito alimentario de Equus conversidens (Perissodactyla, Equidae) del Pleistoceno Tardío (Rancholabreano) de Hidalgo, centro de México: Revista Mexicana de Ciencias Geológicas, 28, 65-82.

Brookes, J., 1828, A Catalogue of the Anatomical and Zoological Museum of Joshua Brookes, Esq. F.R.S. F.L.S. \&c., 124 p.

Carbot-Chanona, G., Gómez-Pérez, L.E., 2014, Adición al registro de megamamíferos del Pleistoceno tardío de Chiapas, México, Memorias del II Simposio de Paleontología en el Sureste de México, 24.

Carbot-Chanona, G., Lagunas-Rodríguez, Z., Jiménez-Moreno, F.J., Suárez, S., 2018, Aspectos paleobiológicos de dos ejemplares de Mammuthus columbi (Mammalia, Proboscidea, Elephantidae) del Pleistoceno de Puebla, centro de México: Boletín de la Sociedad Geológica Mexicana, 69(3), 591-609.

Carbot-Chanona, G., Vázquez-Bautista, D., 2006, Presencia de Bison en Chiapas, México, in X Congreso Nacional de Paleontología, Universidad Nacional Autónoma de México, Instituto de Geología, Publicación Especial 5, Universidad Nacional Autónoma de México, $96 \mathrm{p}$.

Castaños, J., Castaños, P., Murelaga, X., AlonsoOlazabal, A., 2012, Kiputz Ix: Un Conjunto Singular de Bisonte Estepario (Bison priscus Bojanus, 1827) Del Pleistoceno Superior De La Península Ibérica: Ameghiniana, 49, 247-261.

Ceballos, G., Arroyo-Cabrales, J., 2012, Lista actualizada de los mamíferos de México 2012: Revista Mexicana de Mastozoología 
Nueva época, 2, 27-80.

Ceballos, G., Arroyo-Cabrales, J., Ponce, E., 2010,

Effects of Pleistocene environmental changes on the distribution and community structure of the mammalian fauna of Mexico: Quaternary Research, 73, 464-473.

Clark, P., Dyke, A., Shakun, J., Carlson, A., Clark, J., Wohlfarth, B., Mitrovica, J., Hostetler, S., McCabe, M., 2009, The Last Glacial Maximum: Science, 325, 710-714.

Cope, E.D., 1884, The Extinct Mammalia of the Valley of Mexico: Proceedings of the American Philosophical Society, 22, 1-21.

Danowitz, M., Hou, S., Mihlbachler, M., Hastings, V., Solounias, N., 2016, A combinedmesowear analysis of late Miocene giraffids from North Chinese and Greek localities of the Pikermian Biome: Palaeogeography, Palaeoclimatology, Palaeoecology, 449, 194-204.

Díaz-Sibaja, R., García-Zepeda, M.L., LópezGarcía, J.R., Tejeda-Alvarado, F., MarínLeyva, A.H., Gutiérrez-Bedolla, M., 2012, Bisontes (Bison) del Rancholabreano de la Cinta-Portalitos y la Piedad-Santa Ana, Michoacán-Guanajuato, en Libro de Resúmenes Del XI Congreso Nacional de Mastozoología, 212 p.

Dillehay, T.D., 1974, Late Quaternary bison population changes on the southern plains: Plains Anthropologist, 19, 180-196.

Dugès, A., 1894, Felis fósil de San Juan de los Lagos, La naturaleza: periódico científico del Museo Nacional de Historia Natural y de la Sociedad Mexicana de Historia Natural, 2, 421-423.

Elder, W.H., 1970, Morphometry of elephant tusks: Zoologica Africana, 5, 143-159.

Falconer, H., 1858, On the species of Mastodon and Elephant occurring in the fossil state in England.-Part II. Elephas: Quarterly Journal of the Geological Society, 14, 81-84.

Ferrusquía-Villafranca, I., 1976, Estudios Geológico-Paleontológicos en la Región Mixteca, Parte 1: Geología del Área
Tamazulapan-Teposcolula-Yanhutlán, Mixteca Alta, Estado de Oaxaca, México, Boletín del Instituto de Geología Universidad Nacional Autónoma de México, 97, 160 p.

Fernández-Jalvo, Y., Andrews, P., 2003, Experimental effects of water abrasion on bone fragments: Journal of Taphonomy, 1, 147-163.

Fernández-Jalvo, Y., Andrews, P., 2016, Atlas of Taphonomic Identifications, 1001+ Images of Fossil and Recent Mammal Bone Modification: Springer, $359 \mathrm{p}$.

Ferrusquía-Villafranca, I., Arroyo-Cabrales, J., Martínez-Hernández, E., Gama-Castro, J., Ruiz-González, J., Polaco, O.J., Johnson, E., 2010, Pleistocene mammals of Mexico: A critical review of regional chronofaunas, climate change response and biogeographic provinciality: Quaternary International, 217, 53-104.

Fortelius, M., Solounias, N., 2000, Functional Characterization of Ungulate Molars Using the Abrasion-Attrition Wear Gradient: A New Method for Reconstructing Paleodiets: American Museum Novitates, 1-36.

France, D.L., 2009, Human and nonhuman bone identification, a color atlas, CRG Press, Taylor and Francis Group, 734 p.

Frison, G.C., Todd, L.G., 1986, The Colby Mammoth Site: Taphonomy and Archaeology of a Clovis Kill in Northern Wyoming, University of New Mexico Press, Albuquerque, $238 \mathrm{p}$.

Froese, D., Stiller, M., Heintzman, P.D., Reyes, A.V., Zazula, G.D., Soares, A.E.R., Meyer, M., Hall, E., Jensen, B.J.L., Arnold, L.J., Macphee, R.D.E., Shapiro, B., Grayson, D.K., 2017, Fossil and genomic evidence constrains the timing of bison arrival in North America: Proceedings of the National Academy of Sciences of the United States of America, 114, 3457-3462.

Gagliano, S.M., 1967, Occupation Sequence at Avery Island, Coastal Studies Series Number 22, $193 \mathrm{p}$. 
Gray, J.E., 1821, On the natural arrangement of vertebrose animals, London Medical Repository: Monthly Journal, and Review, 15(88), 296-310.

Guthrie, R.D., 1966, Bison horn cores: Character choice and systematics: Journal of Paleontology, 40(3) 738-740.

Guthrie, R.D., 1970, Bison evolution and zoogeography in North America during the Pleistocene: The Quarterly Review of Biology, 45, 1-15.

Gutiérrez Bedolla, M., García-Zepeda, M.L., López-García, R., Arroyo-Cabrales, J., Marín-Leyva, A.H., Meléndez-Herrera, E., Fuentes-Farías, A.L., 2016, Diet and habitat of Mammuthus columbi (Falconer, 1857) from two Late Pleistocene localities in central western Mexico: Quaternary International, 406, 137-146.

Hamilton-Smith, G., 1827, Supplement to the order Ruminantia, en Griffith, E., HamiltonSmith, C., Pidgeon, E. (eds.), The Class Mammalia arranged by the Baron Cuvier, with specific descriptions, The Animal Kingdom arranged in conformity with its organization, By the Baron Cuvier, Member of the Institute of France, with additional descriptions of all the species hiterto named, and of many not before noticed, Volume the Fourth, Geo. B. Whittaker, 498 p.

Harlan, R., 1825, Fauna Americana: beign a description of the mammiferous animals inhabiting North America, Anthony Finley, $318 \mathrm{p}$.

Hayek, L., Bernor, R., Solounias, N., 1992, Preliminary studies of hipparionine horse diet as measured by tooth microwear: Annales Zoologici, 28, 187-200.

Haynes, G.V., 2008, Younger Dryas "black mats" and the Rancholabrean termination in North America: Proceedings of the National Academy of Sciences of the United States of America, 105, 6520-6525.

Haynes, C.V., 1985, Mastodon-bearing springs and late Quaternary geochronology of the
Lower Pomme de Terre Valley, Missouri: Geological Society of America Special Paper, 204, 1-35.

Hodgson, J.A., Allmon, W.D., Nester, P.L., Sherpa, J.M., Chiment, J.J., 2008, Comparative osteology of late Pleistocene mammoth and mastodon remains from the Watkins Glen site, Chemung County, New York, en Allmon, W.D., Nester, P.L. (eds.), Mastodon Paleobiology, Taphonomy, and Paleoenvironment in the Late Pleistocene of New York State: Studies on the Hyde Park, Chemung, and North Java Sites, Palaeontographica Americana, 301-367.

Illigeri, C.D., 1811, Prodromus systematis mammalium et avium additis terminis zoographicis utriusque classis, eorumque versione germanica, Salfeld, Berolini, 301 p. Jiménez-Hidalgo, E., Cabrera-Pérez, L., MacFadden, B.J., Guerrero-Arenas, R., 2013, First record of Bison antiquus from the Late Pleistocene of southern Mexico: Journal of South American Earth Sciences, 42, 83-90. Jiménez-Hidalgo, E., Campos-Camacho, M.A., Guerrero-Arenas, R., Cabrera-Pérez, L., 2012, The late Pleistocene equids from northwestern Oaxaca, southern Mexico, in $108^{\text {th }}$ Annual Meeting, Geological Society of America. Geological Society of America Abstracts with Programs, 44(3), 10 p.

Jiménez-Hidalgo, E., Guerrero-Arenas, R., Macfadden, B.J., Cabrera-Pérez, L., 2011, The late pleistocene (Rancholabrean) Viko vijin local fauna from la Mixteca alta, North Western Oaxaca, Southern Mexico: Revista Brasileira de Paleontologia, 14, 15-28.

Khan, M.A., Kostopoulos, D.S., Akhtar, M., Nazir, M., 2010, Bison remains from the Upper Siwaliks of Pakistan: Neues Jahrbuch für Geologie und Paläontologie - Abhandlungen, 258, 121-128.

Klaus-Dieter, B., Sack, W.O., Röck, S., 2009, Anatomy of the Horse, Fifth revised edition, Germany, Schlütersche Verlagsgesellschaft, $199 \mathrm{p}$. 
Kurtén, B., 1968, Pleistocene mammals of Europe, Aldine Transaction, $317 \mathrm{p}$.

Linneo, C., 1758, Systema Naturae per Regna Tria Naturae, Secundum Classes, Ordines, Genera, Species, cum Characteribus, Differentiis, Synonymis, Locis. Tomus I. Editio Decima, Reformata, Laurentii Salvii, $824 \mathrm{p}$.

Lucas, S.G., 2008, Late Cenozoic Vertebrate Fossil Assemblages From Jalisco, Mexico: New Mexico Museum of Natural History and Science Bulletin, 44, 51-64.

Marín-Leyva, A.H., DeMiguel, D., GarcíaZepeda, M.L., Ponce-Saavedra, J., ArroyoCabrales, J., Schaaf, P., Alberdi, M.T., 2016, Dietary adaptability of Late Pleistocene Equus from West Central Mexico: Palaeogeography, Palaeoclimatology, Palaeoecology, 441, 748-757.

McCuaig-Balkwill, D., Gumbaa, S.L., 1992, A guide to the identification of postcranial bones in Bos taurus and Bison bison, Canadian Museum of Nature, Syllogeus No. 71, 277 p. McDonald, J.N., 1981, North American bison: their classification and evolution. University of California Press, 316 p.

Miller, I.M., Pigati, J.S., Scott Anderson, R., Johnson, K.R., Mahan, S.A., Ager, T.A., Baker, R.G., Blaauw, M., Bright, J., Brown, P.M., Bryant, B., Calamari, Z.T., Carrara, P.E., Cherney, M.D., Demboski, J.R., Elias, S.A., Fisher, D.C., Gray, H.J., Haskett, D.R., Honke, J.S., Jackson, S.T., Jimenez-Moreno, G., Kline, D., Leonard, E.M., Lifton, N.A., Lucking, C., Gregory McDonald, H., Miller, D.M., Muhs, D.R., Nash, S.E., Newton, C., Paces, J.B., Petrie, L., Plummer, M.A., Porinchu, D.F., Rountrey, A.N., Scott, E., Sertich, J.J.W., Sharpe, S.E., Skipp, G.L., Strickland, L.E., Stucky, R.K., Thompson, R.S., Wilson, J., 2014, Summary of the Snowmastodon Project Special Volume. A high-elevation, multi-proxy biotic and environmental record of MIS 6-4 from the Ziegler Reservoir fossil site, Snowmass
Village, Colorado, USA: Quaternary Research, 82, 618-634.

Olsen, S.J., 1972, Osteology for the Archeologist. No. 3 the American Mastodon and the Woolly Mammoth, Papers of the Peabody Museum of Archaeology and Ethnology, Harvard University, 56, 1-45.

Olsen, S.J., 1960, Post-cranial skeletal characters of Bison and Bos, Papers of the Peabody Museum of Archaeology and Ethnology, Harvard University, 35(4), 1-15.

Osborn, H.F., 1942, Proboscidea. A Monograph of the Discovery, Evolution, Migration and Extinction of the Mastodonts and Elephants of the World: Vol. II Stegodontoidea, Elephantoidea, The American Museum Press, American Museum of Natural History, $1675 \mathrm{p}$.

Osborn, H.F., 1905, Recent vertebrate paleontology. Fossil mammals of Mexico: Science, 21, 931-932.

Owen, R., 1848, Description of Teeth and portions of Jaws of two extinct Anthracotheroid Quadrupeds (Hyopotamus vectianus and Hyopbovinus) discovered by the Marchioness of Hastings in the Eocene Deposits on the N. W. coast of the Isle of Wight: with an attempt to develope Cuvier's idea of the Classification of Pachyderms by the Number of their Toes: Quarterly Journal of the Geological Society of London, 4, 103-141.

Owen, R., 1869, On Fossil Remains of Equines from Central and South America Referable to Equus Conversidens, Ow., Equus tau, Ow., and Equus arcidens, Ow.: Philosophical Transactions of the Royal Society of London, 159, 559-573.

Pales, L., Garcia, M.A., 1981, Atlas Ostéologique pour servir á I’identification des Mammiféres du Quaternaire. II. Tête - Rachis Ceintures scapulaire et pelvienne. Membres. Herbivores, Paris, Editions du Centre National de la Recherche Scientifique, 263 p. Pérez-Crespo, V.A., Arroyo-Cabrales, J., SantosMoreno, A., 2008, Generalidades de los 
Mamíferos del Pleistoceno Tardío de Oaxaca:

Naturaleza y Desarrollo, 6, 5-11.

Prat, F., Delpech, F., Cancel, N., Guadelli, J.-L., Slott-Moller, R., 2010, Le Bison des steppes, Bison priscus Bojanus, 1827, de la grotte d'Habarra à Arudy (Pyrénées-Atlantiques), PALEO: Revue d'archéologie préhistorique, $15,1-115$.

Priego-Vargas, J., Bravo-Cuevas, V.M., JiménezHidalgo, E., 2016, The record of Cenozoic horses in Mexico: current knowledge and palaeobiological implications: Palaeobiodiversity and Palaeoenvironments, 96, 305-331.

Saarinen, J., Karme, A., Cerling, T., Uno, K., Säilä, L., Kasiki, S., Ngene, S., Obari, T., Mbua, E., Manthi, F.K., Fortelius, M., 2015, A New Tooth Wear-Based Dietary Analysis Method for Proboscidea (Mammalia): Journal of Vertebrate Paleontology, 35(3), e918546-1- e918546-8.

Sánchez-Rojas, L.E., Aranda-Osorio, J.N., Zárate-López, J., Castro-Rodríguez, M.G., 2000, Carta geológico-minera. Zaachila E14-12, Oaxaca, Escala 1:250,000, Servicio Geológico Mexicano.

Savage, D.E., 1951, Late Cenozoic vertebrates of the San Francisco Bay region, University of California publications: Bulletin of the Department of Geological Sciences, 28(10), 215-314.

Schlesinger, W.H., 1982, Carbon Storage in the Caliche of Arid Soils: Soil Science, 133(4), 247-255.

Schmid, E., 1972, Atlas of animal bones. For prehistorians, archaeologists and Quaternary geologists, Elsevier Publishing Company, 159 p.

Scott, G.R., Lindvall, R.M., 1970, Geology of new occurrences of Pleistocene bisons and peccaries in Colorado: US Geological Survey Professional Paper, 700, B141-B149.

Scott, W.E., Pierce, K.L., Bradbury, J.P., Forester, R.M., 1982, Revised Quaternary stratigraphy and chronology in the American Falls Area, Southeastern Idaho: Idaho Bureau of Mines and Geology Bulletin, 581-595.

Shapiro, B., Drummond, A.J., Rambaut, A., Wilson, M.G., Matheus, P.E., Sher, A. V, Pybus, O.G., Gilbert, M.T.P., Barnes, I., Binladen, J., Willerslev, E., Hansen, A.J., 2004, Rise and Fall of the Beringian Steppe Bison: Science, 306, 1561-1565.

Shpansky, A.V., Svyatko, S.V., Reimer, P.J., Titov, S.V., 2016, Records of Bison priscus Bojanus (Artiodactyla, Bovidae) skeletons in Western Siberia: Russian Journal of Theriology, 15(2), 100-120.

Skinner, M.F., Kaisen, O.C., 1947, The fossil Bison of Alaska and preliminary revision of the genus: Bulletin of the American Museum of Natural History, 89(3), 123-256.

Solorzano, F., 2002, Localidades y fauna fósil registrada para el Estado de Jalisco, Reporte técnico del Instituto Nacional de Antropología e Historia, Jalisco, 9 p.

Solounias, N., Moelleken, S.M.G., 1994, Differences in diet between two archaic ruminant species from Sansan, France: Historical Biology, 7(3), 203-220.

Solounias, N., Semprebon, G.M., 2002, Advances in the Reconstruction of Ungulate Ecomorphology with Application to Early Fossil Equids: American Museum Novitates, 3366, 1-49.

Stevens, A.M., 1978, Sexual Dimorphism in some Post-cranial Elements of Bison latifrons, Los Angeles, California, California State University, tesis de maestría.

Todd, L.C., 1987, Appendix 1: Bison bone measurements, en Frison, G.C., Todd, L.C. (eds.), The Horner Site. The Type Site of the Cody Cultural Complex, Academic Press, Inc., $371-403$.

Todd, L.G., Hofman, J.L., Schultz, G.B., 1992, Faunal Analysis and Paleoindian Studies: Reexamination of the Lipscomb Bone Bed: Plains Anthropologist, 37(139), 137-165. 
Ungar, P., 2002, Microware software, Version 4.02. A semi-automated image analysis system for the quantification of dental microwear.

Villada, M.M., 1903, Acerca de la fauna fósil del valle de México: Anales del Museo Nacional de México, primera época (1877-1903), 7, 441-451.

Voorhies, M.R., 1969, Taphonomy and population dynamics of an early Pliocene vertebrate fauna, Knox County, Nebraska, University of Wyoming: Contributions to Geology, Special Paper no. 1, 1-69.

Wilson, M.C., Hills, L.V, Shapiro, B., 2008, Late Pleistocene northward-dispersing Bison antiquus from the Bighill Creek Formation, Gallelli Gravel Pit, Alberta, Canada, and the fate of Bison occidentalis: Canadian Journal of Earth Sciences, 45, 827-859. 